\section{ORIGINAL} RESEARCH

J. Sedlacik

U. Löbel

M. Kocak

R.B. Loeffler

J.R. Reichenbach

A. Broniscer

Z. Patay

C.M. Hillenbrand

\title{
Attenuation of Cerebral Venous Contrast in Susceptibility-Weighted Imaging of Spontaneously Breathing Pediatric Patients Sedated with Propofol
}

BACKGROUND AND PURPOSE: SWI is known for its detailed visualization of the cerebral venous system and seems to be a promising tool for early detection of cerebrovascular pathologies in children, who are frequently sedated for MR imaging. Because sedation influences cerebral hemodynamics, we hypothesized that it would affect cerebral venous contrast in SWI.

MATERIALS AND METHODS: SWI (125 examinations) of 26 patients (age, 2-16 years) was reviewed in this study. Images were acquired of patients sedated with propofol. Reviewers classified the images by weak or strong venous contrast. Physiologic data, such as etCO $\mathrm{CO}_{2} \mathrm{BP}$, age, and CBF by arterial spin-labeling, were monitored and collected during MR imaging. A generalized estimating equation approach was used to model associations of these parameters with venous contrast.

RESULTS: $\mathrm{EtCO}_{2}$ and $\mathrm{CBF}$ were found to correlate with venous contrast, suggesting that patients with high et $\mathrm{CO}_{2}$ and $\mathrm{CBF}$ have weak contrast and patients with low etCO $\mathrm{C}_{2}$ and $\mathrm{CBF}$ have strong contrast. $\mathrm{BP}$ was also found to correlate with the venous contrast of SWI, suggesting that patients with high BP have strong venous contrast. No significant correlations were found for any other physiologic parameters.

CONCLUSIONS: We found that the venous contrast in SWI is affected by propofol sedation in spontaneously breathing patients. We also found that low et $\mathrm{CO}_{2}$, low CBF, and high $\mathrm{BP}$ are associated with strong venous contrast. Reviewing SWI data in light of physiologic measures may therefore help prevent potential misinterpretations of weak venous contrast in SWI examinations under propofol sedation.

ABBREVIATIONS: $\mathrm{ASL}=$ arterial spin-labeling; $\mathrm{BOLD}=$ blood oxygenation level-dependent; $\mathrm{BP}=$ blood pressure; $\mathrm{CBF}=$ cerebral blood flow; et $\mathrm{CO}_{2}=$ end-tidal carbon dioxide; $\mathrm{FA}=$ flip angle; $\mathrm{GEE}=$ generalized estimating equations; $\mathrm{PET}=$ positron-emission tomography; O2TIPS = quantitative imaging of perfusion using single subtraction, second version, with thin-section $\mathrm{TI}_{1}$ periodic saturation; $\mathrm{RBC}=$ red blood cell count; $\mathrm{RR}=$ respiration rate; $\mathrm{SWI}=$ susceptibility-weighted imaging

$\mathbf{S}^{\mathrm{u}}$ usceptibility-weighted imaging reveals the cerebral venous system with great anatomic detail. ${ }^{1,2}$ The signal intensity, which determines the contrast of venous vessels in SWI, depends on the deoxyhemoglobin concentration in the blood and is referred to as the BOLD signal intensity. ${ }^{3}$ Under normal physiologic conditions, cerebral venous blood has an oxygenation level of $\sim 50 \%,{ }^{4}$ and venous vessels appear hy-

Received August 14, 2009; accepted after revision October 15.

From the Departments of Radiological Sciences (J.S., U.L., R.B.L., Z.P., C.M.H.), Oncology (A.B.), and Biostatistics (M.K.), St. Jude Children's Research Hospital, Memphis, Tennessee; and Medical Physics Group (J.R.R.), Institute for Diagnostic and Interventional Radiology, University Clinics, Friedrich Schiller University, Jena, Germany.

This study was supported by American Lebanese Syrian Associated Charities (ALSAC) and in part by the German Academic Exchange Service (DAAD, Grant PPP-D/06/29424).

Previously presented in part at: American Society of Neuroradiology 47th Annual Meeting, Vancouver, British Columbia, Canada, May 16-21, 2009; and the International Society for Magnetic Resonance in Medicine, 17th Scientific Meeting and Exhibition, April 18-24, 2009.

Please address correspondence to Claudia Hillenbrand, St. Jude Children's Research Hospital, 262 Danny Thomas PI, Mail Stop 220, Memphis, TN 38105; e-mail: claudia. hillenbrand@stjude.org

Indicates open access to non-subscribers at www.ajnr.org

DOI 10.3174/ajnr.A1960 pointense on SWI, with good contrast to their surrounding brain tissue. Variations of the BOLD signal intensity depend mainly on changes in the $\mathrm{CBF}$. With increased global CBF due to carbogen $\left(95 \% \mathrm{O}_{2}, 5 \% \mathrm{CO}_{2}\right)$ inhalation, ${ }^{5}$ the venous blood oxygenation level is increased, causing a higher venous BOLD signal intensity. Therefore, cerebral veins appear almost isointense in SWI, and their contrast to the surrounding tissue vanishes. ${ }^{6}$ On the other hand, CBF can be easily decreased globally by, for example, common doses of caffeine, ${ }^{7}$ decreasing venous blood oxygen saturation, lowering BOLD signal intensity, and thereby increasing venous contrast in SWI. ${ }^{8}$ Detection of neuronal activation is also commonly achieved by repeated measurements of BOLD signal intensity changes. Here, CBF in activated areas increases, exceeding the actual demand for oxygen of the activated neurons, ${ }^{9}$ resulting in a higher blood oxygenation level and consequently a higher BOLD signal intensity. ${ }^{10}$

SWI is a powerful diagnostic tool for early detection and better characterization of cerebrovascular malformations, hemorrhages, iron deposits, and calcifications in children. ${ }^{11}$ However, MR imaging examinations of very young children are frequently performed under sedation by using the common anesthetic propofol. ${ }^{12,13}$ It was shown by a ${ }^{15} \mathrm{O}-\mathrm{PET}$ 


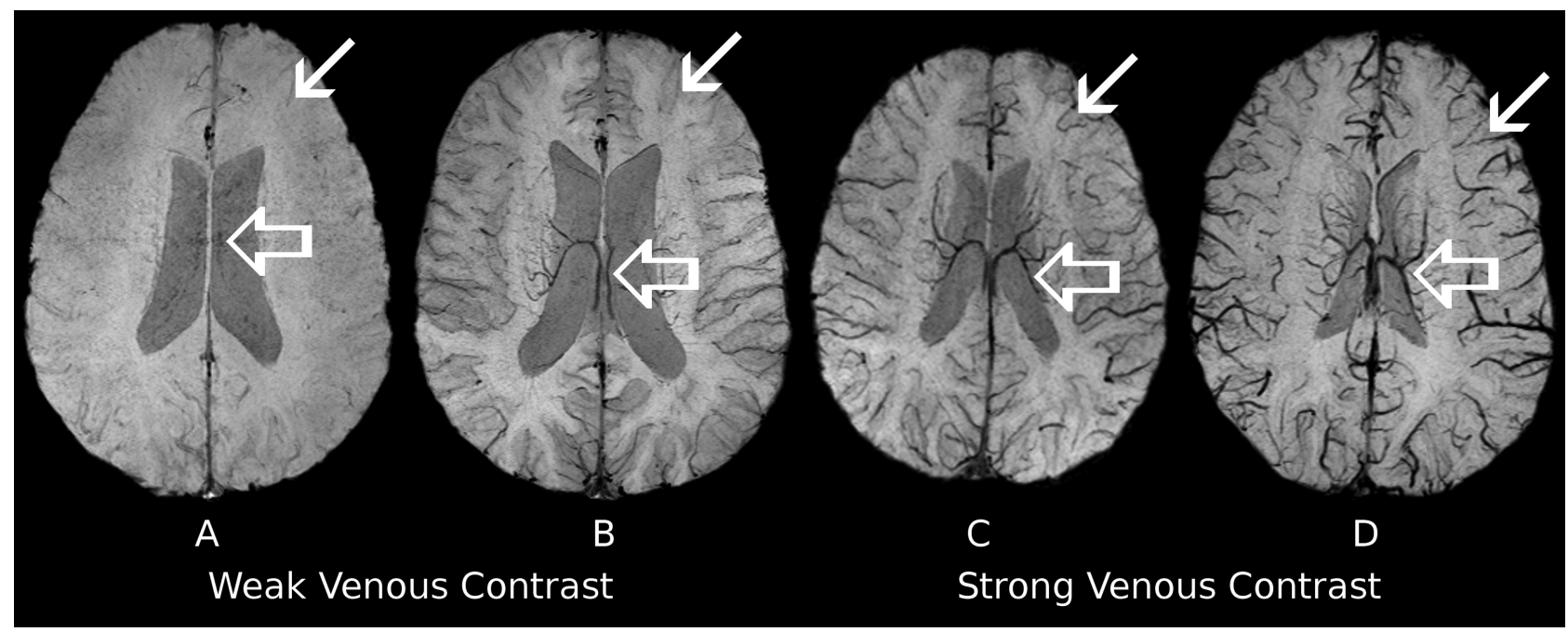

Fig 1. Example of SWI data demonstrating venous contrast classification. All images were generated and visualized by using identical parameters. Examinations with obviously vanished veins $(A)$ as well as examinations showing missing contrast of cortical veins (thin arrow) while maintaining intermediate contrast of deep veins (thick arrow) (B) were categorized as weak venous contrast. Examinations showing strong contrast of deep veins while maintaining intermediate contrast of cortical veins $(C)$ and examinations with obviously strong venous contrast for all veins $(D)$ were categorized as strong venous contrast.

study in propofol-sedated healthy men ${ }^{14}$ that cerebral metabolism and CBF were comparably decreased, keeping the oxygen extraction fraction constant. Therefore, venous blood oxygen saturation and BOLD signal intensity should not be directly affected due to propofol sedation. However, $\mathrm{CO}_{2}$ reactivity of cerebral vessels and autoregulation of brain perfusion are retained under propofol sedation. ${ }^{15-18}$ As pediatric patients breathe spontaneously during sedation while undergoing MR imaging, their respiration is depressed by propofol. ${ }^{19}$ Therefore, propofol indirectly increases the partial pressure of $\mathrm{CO}_{2}$ in the arterial blood, causing cerebral vessel dilation, ${ }^{20}$ leading to an increase in $\mathrm{CBF}^{21}$ and a consequent rise in the BOLD signal intensity, which in turn attenuates the contrast of cerebral veins on SWI. ${ }^{6}$ This effect was recently observed in a limited number of cases without sufficient evidence. $^{22}$ Although the authors hypothesized that this finding could be caused by a change of cerebral metabolism and arterial oxygen saturation due to sedation, they were not able to prove or refute this. Nevertheless, a correlation between sedation and venous contrast in SWI is likely, and this relationship needs to be better understood to allow correct interpretation of SWI data. Even though it is not anticipated that prominent (ie, clinically relevant) pathologies such as arteriovenous malformations ${ }^{23,24}$ or Sturge-Weber syndrome ${ }^{25,26}$ would be missed if venous contrast were attenuated due to propofol sedation, it is possible that the exact extent or degree of the disease could be underestimated. On the other hand, with SWI becoming more widely available, it is conceivable that different levels of venous contrast might be used in the future to better differentiate diagnoses or to stage diseases, as recently presented by Newbern et $\mathrm{al}^{27}$ at the 2009 annual meeting of the American Society of Neuroradiology.

Therefore, the purpose of this study was to investigate the effect of sedation with propofol on venous contrast in SWI. We hypothesized that physiologic measures such as blood pressure, heart rate, respiration rate, and etCO $\mathrm{C}_{2}$, which reflect the effect of sedation on respiration and the cardiovascular system, would correlate with the apparent contrast of cerebral veins in SWI. We thereby assumed that other physiologic parameters such as patient age or time of treatment should not correlate with the venous contrast in SWI images exhibiting healthy brain tissue. Testing these hypotheses should help to prevent potential misinterpretation of SWI images acquired under sedation.

\section{Materials and Methods}

SWI data were collected from 26 patients (14 girls, 12 boys; age at diagnosis, 2-16 years) who were enrolled between May 2006 and February 2009 in an institutional review board-approved clinical phase I trial at our institution. The patients had diagnoses of diffuse pontine glioma and were being treated with local radiation therapy to the brain stem $(n=25)$ or to the whole brain $(n=1)$. Radiation was fractionated and administered over 6 weeks ( 30 fractions, 54 Gy total dose) ${ }^{28}$ During the study, patients received vandetanib (Zactima; ZD6474), an antiangiogenic drug. ${ }^{29}$ A secondary objective of the phase I trial was to assess vascular changes during therapy by advanced MR imaging techniques. SWI images were acquired on a 3T clinical MR imaging scanner (Magnetom Trio; Siemens Medical Solutions, Erlangen, Germany). Imaging parameters were: TE/TR/FA, $25 \mathrm{~ms} / 56 \mathrm{~ms} / 20^{\circ}$; FOV, $230 \times 115 \times 144 \mathrm{~mm}^{3}$; matrix, $512 \times 254 \times$ 72 ; voxel size, $0.45 \times 0.45 \times 2 \mathrm{~mm}^{3}$; parallel imaging acceleration factor, 2. Six consecutive scans were acquired in 14 patients, and 12 patients received from 1 to $5 \mathrm{MR}$ imaging examinations in our study. Follow-up examinations were performed about 2 weeks and about 1 , 2,4 , and 6 months after the first scan. In total, 125 SWI examinations covering the whole brain were rated in consensus by 2 reviewers. The venous contrast in SWI was classified in 2 groups: group 1 showing good to strong contrast and group 2 showing weak to almost no venous contrast between cerebral veins and parenchyma (Fig 1). The characteristic criterion for strong contrast was good delineation of cortical venous vessels. Examinations with missing contrast of cortical veins while maintaining intermediate deep venous vessel contrast were categorized as weak venous contrast.

As confirmed by radiation therapy dose maps, inferior parts of the brain (ie, medulla oblongata, brain stem, and cerebellum) were subject to high doses, making radiation-induced vasculopathy likely. 


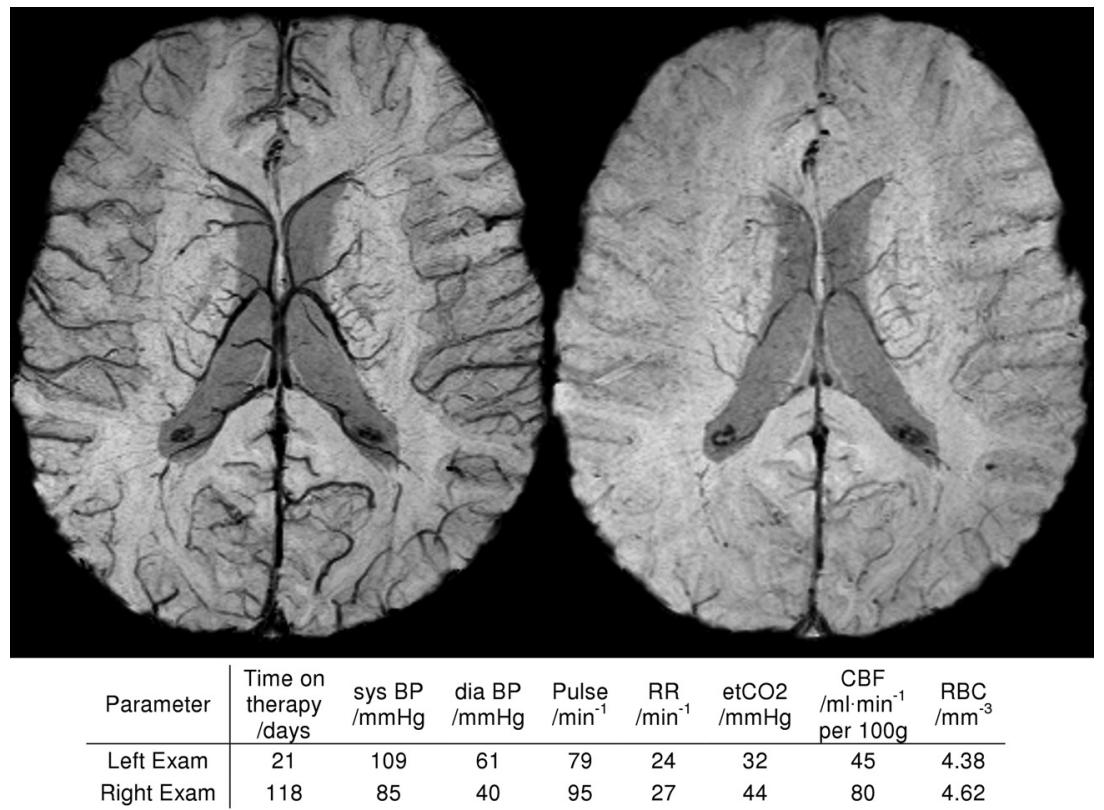

Fig 2. Example SWI data of a 7-year-old patient on 2 different examination dates to demonstrate the intrasubject variation of venous contrast. The patient was sedated for both MR imaging examinations. Physiologic measures (BP, $\mathrm{RR}$, etCO $, \mathrm{CBF}, \mathrm{RBC}$ ) at both examinations are shown for comparison. The images were generated and visualized by using identical parameters.

Therefore, only veins of the cerebrum were used for evaluation to minimize any compromising effect of high radiation doses.

The complete set of diagnostic images of the patient who received radiation to the whole brain was carefully reviewed for abnormal features that might be related to radiation therapy. Because no abnormalities were evident, this patient's SWI examinations were not excluded from our study.

One patient presented with supratentorial and infratentorial tumor dissemination via CSF (leptomeningeal disease) at the time of the fourth MR imaging examination. Because of this, the patient was taken off the clinical trial and his last examination was also excluded from further evaluation in our study, because it is possible that leptomeningeal tumor spread may compromise venous contrast on SWI through compromising venous outflow and causing congestion within transmedullary veins.

Of the 125 SWI scans performed, 114 were acquired under sedation with the patient breathing spontaneously. Eleven examinations were not performed with the patient under sedation and were not formally evaluated. Sedation was maintained by infusion of propofol $(150-300 \mu \mathrm{g} / \mathrm{kg} / \mathrm{min})$. The individual doses were adjusted by the anesthesiologists to allow a smooth MR imaging examination with minimal risk for the patient. Due to different individual sensitivities to the anesthetic, the actual dose of propofol was not correlated with venous contrast. We instead investigated physiologic measures, which are potential measures of the actual effect of propofol on the subject. During the SWI scan, the following physiologic parameters were monitored and recorded: pulse, systolic and diastolic BP, respiration rate, and etCO $\mathrm{C}_{2}$. To further investigate whether nonsedation-related physiologic parameters also affect venous contrast in SWI, we correlated patient age at examination and red blood cell count with venous contrast. As an additional measure of the effect of sedation on CBF, ASL perfusion data by using a Q2TIPS sequence was evaluated along with the SWI data. ${ }^{30}$ ASL sequence parameters were: TE/TR, $23 \mathrm{~ms} /$ $2280 \mathrm{~ms} ; \mathrm{TI}_{1} / \mathrm{TI}_{2}, 700 \mathrm{~ms} / 1400 \mathrm{~ms}$; FOV, $210 \times 210 \mathrm{~mm}^{2}$; matrix $=$ $64 \times 64$; section thickness, $5 \mathrm{~mm}$; 11 sections. Quantitative CBF values were calculated for sections located above the anteroposterior commissure line following a method proposed by Wang et al. ${ }^{31}$ The mean $\mathrm{CBF}$ value for gray matter was calculated by using a histogrambased segmentation algorithm (ie, thresholding technique) of the upper brain CBF values.

\section{Statistical Methods}

Logistic regression model ${ }^{32}$ were used to investigate the association of physiologic measurements with venous contrast at single time windows (eg, baseline scans, week 2 scans, and week 4 scans) in a crosssectional fashion to guide the rest of the statistical analyses. In such models, venous contrast was a dichotomous response, and each physiologic variable was investigated independently. After identifying physiologic variables of interest that were associated with venous contrast in at least 1 time window, the investigation was performed in a longitudinal fashion, by using GEE models, ${ }^{33}$ which take into account intrapatient variability as patients have serial examinations over time. In the GEE models, venous contrast in SWI was again a dichotomous response, assuming binomial distribution with a logit link function, which is commonly used for binary data. Because the time intervals between the subsequent examinations were not equally spaced and not identical between patients, a spatial-power covariance structure was used to take into account intrapatient correlations. As this was an exploratory analysis, $P$ values reported were not adjusted for multiplicity, and the results must be considered in the context of an exploratory analysis and should be confirmed with larger prospective studies.

\section{Results}

All of the $11 \mathrm{MR}$ imaging examinations acquired without sedation showed strong venous contrast. Because physiologic data were not monitored during those scans, they were excluded from further statistical analysis. MR imaging examinations under sedation showed weak venous contrast by our classification in 55 SWI scans and strong contrast in 59. As shown in Fig 2, this variation of contrast was observed even in the same subjects on different examination dates. Only 3 pa- 

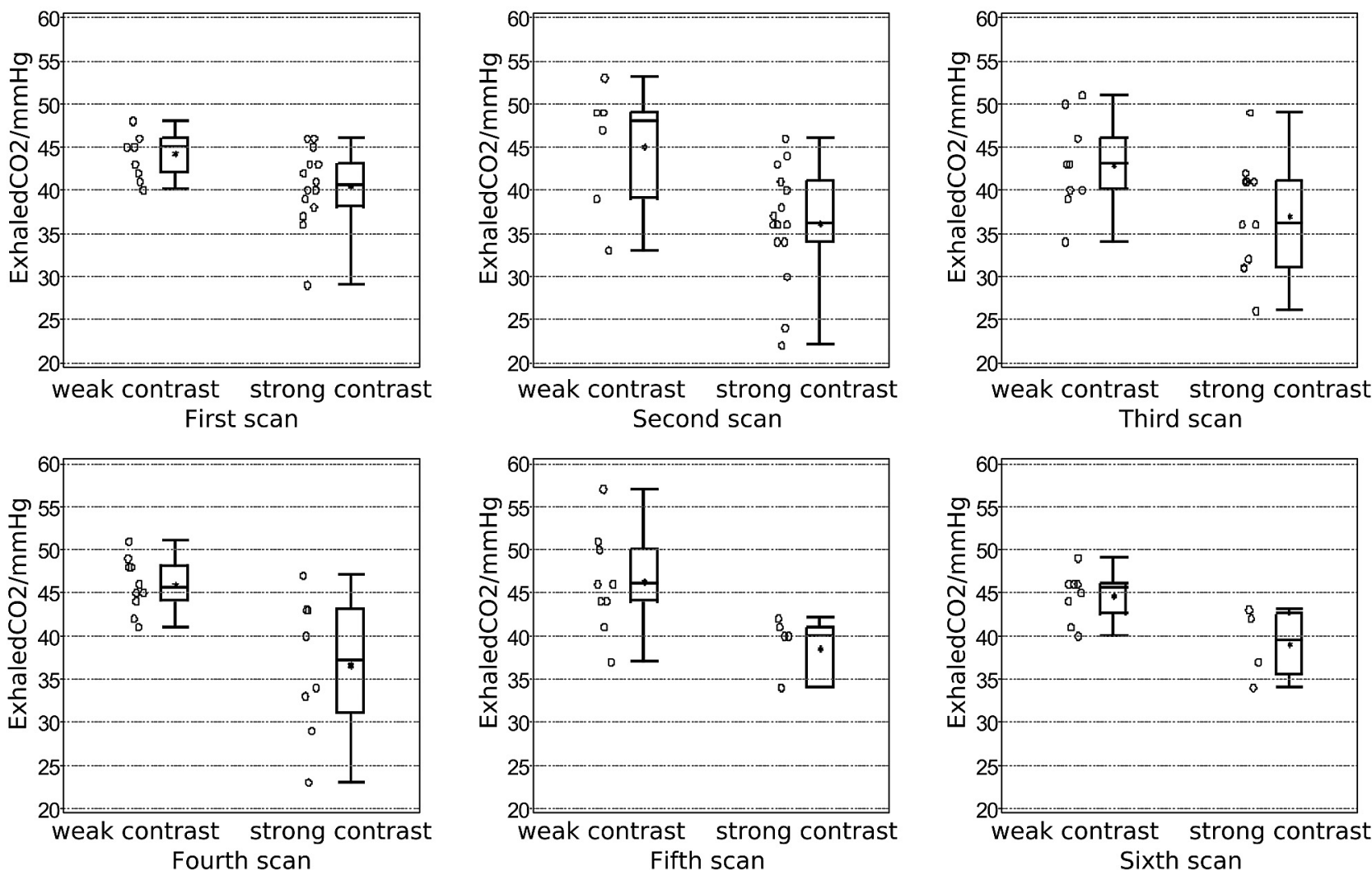

Fig 3. Scatter and boxplots shown for et $\mathrm{CO}_{2}$. Exhaled $\mathrm{CO}_{2}$ is plotted for both groups of different venous contrast in SWI and separately for all 6 time points of consecutive MR imaging examinations. The asterisk within the boxplot denotes the mean value of the particular et $\mathrm{CO}_{2}$ distribution.

\begin{tabular}{|c|c|c|c|c|c|c|}
\hline \multirow[b]{2}{*}{ Parameter } & \multicolumn{6}{|c|}{ Examination } \\
\hline & $\begin{array}{c}\text { First } \\
(n=23)\end{array}$ & $\begin{array}{l}\text { Second } \\
(n=21)\end{array}$ & $\begin{array}{c}\text { Third } \\
(n=21)\end{array}$ & $\begin{array}{l}\text { Fourth } \\
(n=19)\end{array}$ & $\begin{array}{c}\text { Fifth } \\
(n=16)\end{array}$ & $\begin{array}{c}\text { Sixth } \\
(n=13)\end{array}$ \\
\hline $\mathrm{CBF}$ & $0.97(.25)$ & $0.90(.016)^{*}$ & $0.95(.071)^{*}$ & $0.94(.051)^{*}$ & $0.90(.050)^{*}$ & $0.97(.26)$ \\
\hline Systolic BP & $1.03(.29)$ & $1.16(.048)^{*}$ & $1.00(.99)$ & $1.01(.83)$ & $0.93(.25)$ & $0.96(.48)$ \\
\hline Diastolic BP & $1.07(.14)$ & $1.23(.037)^{*}$ & $1.01(.83)$ & $1.08(.14)$ & $1.01(.93)$ & $1.04(.54)$ \\
\hline Pulse & $1.00(.98)$ & $0.99(.70)$ & $0.96(.26)$ & $0.98(.7)$ & $1.03(.49)$ & $0.97(.50)$ \\
\hline $\mathrm{etCO}_{2}$ & $0.72(.050)^{*}$ & $0.8(.036)^{*}$ & $0.84(.066)^{*}$ & $0.68(.062)^{*}$ & $0.65(.071)^{*}$ & $0.60(.089)$ \\
\hline $\mathrm{RR}$ & $1.04(.67)$ & $1.26(.15)$ & $1.07(.49)$ & $1.08(.44)$ & $1.02(.82)$ & $0.81(.26)$ \\
\hline Age & $1.06(.59)$ & $0.93(.54)$ & $0.89(.28)$ & $0.98(.87)$ & $1.15(.26)$ & $1.16(.28)$ \\
\hline RBC & $4.34(.36)$ & $0.42(.49)$ & $0.27(.25)$ & $1.16(.85)$ & $0.67(.71)$ & $2.39(.62)$ \\
\hline
\end{tabular}

Note:-Asterisks denote odds ratios significant to a level of $P<.1$.

tients showed consistently weak venous contrast for all examinations.

Figure 3 shows the variation in etCO $\mathrm{CO}_{2}$ between the 2 contrast groups at all MR imaging examination dates. For etCO $\mathrm{C}_{2}$, a clear trend was observed. The mean and median values were always smaller in the group with good venous contrast. The results of the cross-sectional analysis with logistic regression models for all physiologic measures are summarized in Table 1. The previous visual finding for etCO $\mathrm{CO}_{2}$ (Fig 2) was supported by a significant association of et $\mathrm{CO}_{2}$ at every time point. As also shown in Table 1, segmented gray matter CBF measures were significantly associated with low SWI contrast for the second to the fifth MR imaging examinations. Systolic and diastolic BP showed significance only at the second time point. All other parameters showed no significant association in the cross-sectional analysis and thus were not further evaluated with the GEE models. Systolic BP and gray matter CBF were also excluded from further evaluation because they were highly correlated with diastolic $\mathrm{BP}$ and etCO $\mathrm{C}_{2}$, respectively. As expected, however, gray matter CBF was strongly associated with venous contrast in a univariate GEE model (odds ratio $=$ $0.94, P=.0003)$. Here, the odds ratio suggests that patients with higher gray matter CBF have poorer venous contrast.

The first GEE model (Table 2 ) resulted in a significant $(P<$ .0001 ) odds ratio of 0.75 , which suggests that, for high et $\mathrm{CO}_{2}$ values, strong venous contrast is less likely than weak contrast in SWI. In the second analysis, etCO $\mathrm{C}_{2}$ and diastolic $\mathrm{BP}$ were investigated in one multivariate GEE model, which again led to significant odds ratios of $0.78(P=.0018)$ for etCO $\mathrm{C}_{2}$ and $1.09(P=.0056)$ for diastolic BP. Here, the odds ratio $>1$ implies that patients who presented with higher diastolic BP were more likely to show strong venous contrast in SWI. 


\begin{tabular}{|c|c|c|c|}
\hline & Odds Ratio & $95 \% \mathrm{Cl}$ & $P$ Value \\
\hline \multicolumn{4}{|l|}{ Model 1} \\
\hline $\mathrm{etCO}_{2}$ & 0.75 & $0.65-0.86$ & $<.0001$ \\
\hline Time points & 0.27 & $0.02-4.24$ & .34 \\
\hline \multicolumn{4}{|l|}{ Model 2} \\
\hline etCO & 0.78 & $0.66-0.91$ & .0018 \\
\hline Diastolic BP & 1.09 & $1.03-1.15$ & .0056 \\
\hline Time points & 0.41 & $0.02-10.1$ & .58 \\
\hline
\end{tabular}

Note:-To describe the longitudinal nature of the data, the actual time points of the follow-up MR imaging examinations were taken into account by the GEE models.

Both GEE models showed that venous contrast did not change significantly over treatment time. This observation is important because it implies that there were probably no therapy-induced effects on venous contrast in the cerebrum, which was not directly targeted by $3 \mathrm{D}$ conformal radiation therapy and, thus, received only minor doses. In agreement with this is the finding that the RBC count did not correlate with venous contrast, because any therapy-induced changes in the RBC count would have to be corrected by blood transfusions. ${ }^{34}$ No correlation of venous contrast with patient age was found in our study.

\section{Discussion}

Our analysis of SWI data from sedated patients showed that such imaging studies should be conducted under well-adjusted physiologic conditions to achieve a good venous contrast and to be able to make reliable comparisons between patients or between scans of an individual patient over time. In particular, et $\mathrm{CO}_{2}$ was strongly associated with venous contrast and should be well controlled.

We are confident that the radiation treatment of our patients did not compromise the validity of our study, because the observed associations of venous contrast in SWI with etCO $\mathrm{C}_{2}, \mathrm{CBF}$, and BP were already obvious in the first scan, which was acquired before onset of radiation and antiangiogenic therapy (Table 1, first examination).

We concluded from our pediatric patients (age, 2-16 years), who were sedated during MR imaging, that age-related changes in physiology have no effect on the observed venous contrast in SWI. There is, however, no evidence based on our results that there is an age dependency of venous contrast for healthy nonsedated subjects, in particular, when considering a significant drop in CBF over several decades of life. ${ }^{35}$

Because we argued that sedation affects respiration, it was interesting that no correlation of respiration rate with venous contrast was found. However, respiration rate is not a measure of the actual tidal volume and thus cannot give evidence of the actual depression of respiration. A similar finding was reported by Iwama et al, ${ }^{19}$ who observed a decrease in tidal volume but not in respiration rate while increasing the propofol infusion rate. The only measure of respiration attenuation available to the anesthesiologist is et $\mathrm{CO}_{2},{ }^{36}$ which was found in our study to be strongly associated with the observed venous contrast in SWI. Based on that finding, it might be possible to ensure good venous contrast in SWI by controlling sedation in such a way that etCO ${ }_{2}$ is kept as low as possible. Karsli et $\mathrm{al}^{16,18}$ reported a maximal etCO $\mathrm{C}_{2}$ value of $30-35 \mathrm{mmHg}$ for which $\mathrm{CBF}$ in sedated children is not artificially elevated. This is also in agreement with our findings for $\mathrm{CBF}$, where lower $\mathrm{CBF}$ values were accompanied by lower etCO $\mathrm{C}_{2}$ and good venous contrast. Conversely, higher $\mathrm{CBF}$ was associated with higher etCO $\mathrm{C}_{2}$ and weak venous contrast, which was confirmed by the consistent odds ratios $<1$ for CBF in Table 1 and for the univariate GEE model (see Results). All of these findings are in perfect agreement with our introductory argument and support our hypothesis that et $\mathrm{CO}_{2}$ reflects respiration attenuation due to intravenous propofol sedation and correlates with venous contrast in SWI.

The observed correlation between diastolic BP and venous contrast suggests that higher $\mathrm{BP}$ is associated with good venous contrast, lower etCO $\mathrm{C}_{2}$, and lower $\mathrm{CBF}$, and that lower BP is linked with weak venous contrast, higher et $\mathrm{CO}_{2}$, and higher $\mathrm{CBF}$, which was confirmed by the consistent odds ratios $>1$ for diastolic BP (Tables 1 and 2). This finding is very interesting, because one would expect high $\mathrm{BP}$ to cause high $\mathrm{CBF}$ and low BP to cause low CBF. However, it was shown that the autoregulatory mechanism of brain perfusion works more efficiently for lower etCO $\mathrm{O}_{2}$ than for higher etCO $\mathrm{CO}_{2} \cdot{ }^{37}$ This could explain the contradictory association between $\mathrm{BP}$ and CBF.

Because our ultimate goal is to monitor therapy-induced changes in tumor tissue of pediatric patients, we have to further investigate to what extent sedation compromises CBF or SWI measurements of tumor tissue. Our study showed that respiration attenuation due to propofol sedation can alter $\mathrm{CBF}$ and SWI contrast in healthy brain tissue. Thus, it might be necessary to correct for sedation-induced effects while monitoring tumors. Future studies that involve CBF and SWI measurements should be acquired under controlled physiologic conditions during sedation. It is also conceivable that the observed sedation effect could be used to assess $\mathrm{CO}_{2}$ reactivity as a pathophysiologic marker of tumor vessels. ${ }^{38}$

We are confident that this effect does not degrade the detection of hemorrhages in sedated patients as reported by Tong et $\mathrm{al},{ }^{39}$ because the hemorrhagic contrast is caused by extravascular deoxyhemoglobin and methemoglobin rather than by the presence of intravenous deoxyhemoglobin. Also note that findings of passive functional MR imaging studies $^{40,41}$ might be compromised by the respiratory attenuation of propofol because the magnitude of the BOLD signal intensity responses are most likely lowered due to elevated resting state perfusion of the brain. Therefore, an increased variation over different subjects or multiple examinations of the same subjects is anticipated for quantitative findings such as the magnitude of BOLD signal intensity responses or numbers of activated voxels.

\section{Conclusions}

Based on the results of our analysis, we conclude that it is necessary to acquire SWI data of sedated patients under wellcontrolled physiologic conditions to achieve a good venous contrast and to be able to make reliable comparisons between different patients or follow-up scans. It was found that et $\mathrm{CO}_{2}$ was strongly associated with venous contrast and must be kept $<30-35 \mathrm{mmHg}$, as reported by Karsli et al, ${ }^{16,18}$ to achieve good venous contrast in SWI. If etCO $\mathrm{C}_{2}$ cannot be well controlled in sedated, spontaneously breathing patients, we strongly recommend reviewing SWI data in light of etCO $\mathrm{CO}_{2}, \mathrm{BP}$, and $\mathrm{CBF}$ measures acquired at the same physiologic state to 
prevent potential misinterpretation of venous contrast in SWI.

\section{Acknowledgments}

We thank Dr. George Bikhazi (Department of Anesthesiology, St Jude Children's Research Hospital) for helpful discussion and Nikita Oigbokie (Clinical Investigation, Department of Radiologic Sciences, St Jude) for collecting patient data. The authors are indebted to Dr. Arzu Onar (Department of Biostatistics, St Jude) for critical review of the manuscript, and to David Galloway (Department of Scientific Editing) for editorial review.

\section{References}

1. Haacke EM, Xu Y, Cheng YC, et al. Susceptibility weighted imaging (SWI). Magn Reson Med 2004;52:612-18

2. Reichenbach JR, Venkatesan R, Schillinger DJ, et al. Small vessels in the human brain: MR venography with deoxyhemoglobin as an intrinsic contrast agent. Radiology 1997;204:272-77

3. Ogawa S, Lee TM, Kay AR, et al. Brain magnetic resonance imaging with contrast dependent on blood oxygenation. Proc Natl Acad Sci U S A 1990;87: 9868-72

4. Sedlacik J, Rauscher A, Reichenbach JR. Obtaining blood oxygenation levels from MR signal behavior in the presence of single venous vessels. Magn Reson Med 2007;58:1035-44

5. Kety SS, Schmidt CF. The effects of altered arterial tensions of carbon dioxide and oxygen on cerebral blood flow and cerebral oxygen consumption of normal young men. J Clin Invest 1948;27:484-92

6. Rauscher A, Sedlacik J, Barth M, et al. Nonnvasive assessment of vascular architecture and function during modulated blood oxygenation using susceptibility weighted magnetic resonance imaging. Magn Reson Med 2005;54: 87-95

7. Cameron OG, Modell JG, Hariharan M. Caffeine and human cerebral blood flow: a positron emission tomography study. Life Sci 1990;47:1141-46

8. Sedlacik J, Helm K, Rauscher A, et al. Investigations on the effect of caffeine on cerebral venous vessel contrast by using susceptibility-weighted imaging (SWI) at 1.5, 3 and 7 T. Neuroimage 2008;40:11-18

9. Fox PT, Raichle ME. Focal physiological uncoupling of cerebral blood flow and oxidative metabolism during somatosensory stimulation in human subjects. Proc Natl Acad Sci U S A 1986;83:1140-44

10. Hoge RD, Atkinson J, Gill B, et al. Investigation of BOLD signal dependence on cerebral blood flow and oxygen consumption: the deoxyhemoglobin dilution model. Magn Reson Med 1999;42:849-63

11. Tong KA, Ashwal S, Obenaus A, et al. Susceptibility-weighted MR imaging: a review of clinical applications in children. AJNR Am J Neuroradiol 2008;29: 9-17

12. Mallory MD, Baxter AL, Kost SI. Propofol vs pentobarbital for sedation of children undergoing magnetic resonance imaging: results from the Pediatric Sedation Research Consortium. Paediatr Anaesth 2009;19:601-11

13. Pershad J, Wan J, Anghelescu DL. Comparison of propofol with pentobarbital/ midazolam/fentanyl sedation for magnetic resonance imaging of the brain in children. Pediatrics 2007;120:e629-e636

14. Kaisti KK, Langsjo JW, Aalto S, et al. Effects of sevoflurane, propofol, and adjunct nitrous oxide on regional cerebral blood flow, oxygen consumption, and blood volume in humans. Anesthesiology 2003;99:603-13

15. Fox J, Gelb AW, Enns J, et al. The responsiveness of cerebral blood flow to changes in arterial carbon dioxide is maintained during propofol-nitrous oxide anesthesia in humans. Anesthesiology 1992;77:453-56

16. Karsli C, Wilson-Smith E, Luginbuehl I, et al. The effect of nitrous oxide on cerebrovascular reactivity to carbon dioxide in children during propofol anesthesia. Anesth Analg 2003;97:694-98

17. Van Aken H, Van Hemelrijck J. Influence of anesthesia on cerebral blood flow and cerebral metabolism: an overview. Agressologie 1991;32:303-06
18. Karsli C, Luginbuehl I, Bissonnette B. The cerebrovascular response to hypocapnia in children receiving propofol. Anesth Analg 2004;99:1049-52

19. Iwama H, Nakane M, Ohmori S, et al. Propofol dosage achieving spontaneous breathing during balanced regional anesthesia with the laryngeal mask airway. J Clin Anesth 2000;12:189-95

20. Brian JE, Jr. Carbon dioxide and the cerebral circulation. Anesthesiology 1998;88:1365-86

21. Ashkanian M, Borghammer P, Gjedde A, et al. Improvement of brain tissue oxygenation by inhalation of carbogen. Neuroscience 2008;156:932-38

22. Kesavadas C, Thomas B, Misra S, et al. Attenuation of cerebral veins in susceptibility-weighted MR imaging performed with the patient under general anesthesia. AJNR Am J Neuroradiol 2008;29:e71

23. Essig M, Reichenbach JR, Schad LR, et al. High-resolution MR venography of cerebral arteriovenous malformations. Magn Reson Imaging 1999;17:1417-25

24. Amemiya S, Aoki S, Takao H. Venous congestion associated with developmental venous anomaly: findings on susceptibility weighted imaging. J Magn Reson Imaging 2008;28:1506-09

25. Mentzel HJ, Dieckmann A, Fitzek C, et al. Early diagnosis of cerebral involvement in Sturge-Weber syndrome using high-resolution BOLD MR venography. Pediatr Radiol 2005;35:85-90

26. Juhasz C, Haacke EM, Hu J, et al. Multimodality imaging of cortical and white matter abnormalities in Sturge-Weber syndrome. AJNR Am J Neuroradiol 2007;28:900-06

27. Newbern MS, Jacobson JP, Oyoyo U, et al. Cyanotic heart disease is associated with increased prominence of cerebral deep medullary veins on susceptibility-weighted imaging in children undergoing evaluation for heart transplantation. American Society of Neuroradiology 47th Annual Meeting, Vancouver, British Columbia, Canada, May 16-21, 2009, scientific poster 206, pp. 400-01

28. Broniscer A, Gajjar A. Supratentorial high-grade astrocytoma and diffuse brainstem glioma: two challenges for the pediatric oncologist. Oncologist 2004;9:197-206

29. Wedge SR, Ogilvie DJ, Dukes M, et al. ZD6474 inhibits vascular endothelial growth factor signaling, angiogenesis, and tumor growth following oral administration. Cancer Res 2002;62:4645-55

30. Luh WM, Wong EC, Bandettini PA, et al. QUIPSS II with thin-slice TI1 periodic saturation: a method for improving accuracy of quantitative perfusion imaging using pulsed arterial spin labeling. Magn Reson Med 1999;41:1246-54

31. Wang J, Alsop DC, Li L, et al. Comparison of quantitative perfusion imaging using arterial spin labeling at 1.5 and 4.0 Tesla. Magn Reson Med 2002;48: 242-54

32. Begg CB. Statistical methods in medical diagnosis. Crit Rev Med Inform 1986;1:1-22

33. Lipsitz SR, Laird NM, Harrington DP. Generalized estimating equations for correlated binary data: using the odds ratio as a measure of association. $\mathrm{Bi}$ ometrika 1991;78:153-60

34. Mercadante S, Ferrera P, Villari P, et al. Effects of red blood cell transfusion on anemia-related symptoms in patients with cancer. J Palliat Med 2009;12: $60-63$

35. Biagi L, Abbruzzese A, Bianchi MC, et al. Age dependence of cerebral perfusion assessed by magnetic resonance continuous arterial spin labeling. J Magn Reson Imaging 2007;25:696-702

36. Wu CH, Chou HC, Hsieh WS, et al. Good estimation of arterial carbon dioxide by end-tidal carbon dioxide monitoring in the neonatal intensive care unit. Pediatr Pulmonol 2003;35:292-95

37. Panerai RB, Deverson ST, Mahony P, et al. Effects of $\mathrm{CO}_{2}$ on dynamic cerebral autoregulation measurement. Physiol Meas 1999;20:265-75

38. Packard SD, Mandeville JB, Ichikawa T, et al. Functional response of tumor vasculature to $\mathrm{PaCO}_{2}$ : determination of total and microvascular blood volume by MRI. Neoplasia 2003;5:330-38

39. Tong KA, Ashwal S, Holshouser BA, et al. Hemorrhagic shearing lesions in children and adolescents with posttraumatic diffuse axonal injury: improved detection and initial results. Radiology 2003;227:332-39

40. Gemma M, de Vitis A, Baldoli C, et al. Functional magnetic resonance imaging (fMRI) in children sedated with propofol or midazolam. J Neurosurg Anesthesiol 2009;21:253-58

41. Souweidane MM, Kim KH, McDowall R, et al. Brain mapping in sedated infants and young children with passive-functional magnetic resonance imaging. Pediatr Neurosurg 1999;30:86-92 